\title{
HUMAN BEINGS IN QUANTUM ANTHROPOLOGY: A PARADOX OF THE DISCONTINUOUS EXPERIENCE OF QUANTUM SPACETIME
}

\author{
Radek Trnka \\ This paper is a shortened version of an invited lecture held at the University of Copenhagen \\ (Department of Anthropology) on 28 March 2019.
}

Keywords: quantum social theory, quantum social science, existence, human beings, isomorphism

\section{INTRODUCTION}

Without any doubt, anthropology is a science about man, and consequently we may ask if anthropological research could ever be unrelated to the physicality of human being in the world? We are "made" of matter and energy, and the whole of our world is a world of information. Matter, energy, and information are the basal substances of human beings, and therefore, new scientific discoveries about these substances cannot be neglected. The paradigmatic change in physics caused by the quantum revolution has shown that matter has a different substance than is expected by classical, Newtonian mechanics. These incentives raise new questions that are important for anthropological theory, such as how to understand human beings in the quantum perspective?

\section{Human Beings in a Quantum Perspective}

There are at least two ways of understanding the underlying substrate of human beings. First, with the help of the metaphysics of presence based on classical, Newtonian mechanics, and second, with the help of the metaphysics proposed by quantum mechanics. These two explanations of the nature of human beings work with different understandings of the basal constituents of matter.

To comprehend the basic difference, the nature of quantum microparticles should be explained at the beginning. What character do microparticles have? In contrast to atoms, the substance of quantum microparticles is surprisingly not material. Under real-life conditions, the observer can determine such qualities of a given microparticle as the specific spin, the frequency of oscillation/amplitude, the angular momentum, the magnetic moment, and the electrical charge at the moment of measurement. Simply said, each microparticle is a very small energetic vortex. The non-material substance of quantum microparticles contrasts to the idea of atoms, which were long believed to be the smallest, "hard" constituents of macro-objects. This fundamental shift in the understanding of the character of the constituents of matter also elicits very insistent questions regarding the nature of human beings. Recently, several quantum-based theoretical models of human beings have emerged in anthropology and in social science:

a) the model of Wendt (2015)

human beings are "walking wave functions" - the realist claim (not an analogy, nor a metaphor) 
b) the model of Trnka and Lorencova (2016)

human beings are quantum systems - a functional analogy (not a metaphor)

Human beings consist of microparticles that show a coherence, enabling the maintenance of the continuity of identity during one's life. Human bodies are made of microparticles, and therefore, we may expect that such a system built from microparticles may also exhibit some behaviors that are similar to the behaviors of microparticles.

c) the model of Russell (2013)

human beings are embodiments of a part of the energy-information field - a functional analogy (not a metaphor)

This model assumes that there is some elemental energy-information field or essence that is constantly present in the world. Some part can manifest itself from this field through matter, for example, in the form of a concrete human being. On one hand, there is energy-information as potentiality, on the other hand, there is the embodiment - as the actualization of potentiality and probability.

The quantum-based models of human beings have several problems. First, we can ask if the principles that originated in quantum mechanics can be also applied in anthropology? The complexities of microparticles and human beings are really very different. So, is it legitimate to apply quantum concepts to human behavior? Second, quantum mechanics and anthropology differ in the understanding of the permanence of existence. In anthropology, it is assumed that the existence of human beings is relatively permanent throughout human life. In contrast, according to quantum mechanics, the existence of microparticles is only transient and dependent on the act of observation.

\section{Differences in the Complexity of Human Beings and Microparticles}

The realist claim assuming human beings to be walking wave functions raises several issues (Allan, 2018; Arfi, 2018; Burgess, 2018; Kessler, 2018). For example, can a physicalist congruence between humans and particles be expected (Michel, 2018)? Humans are definitely much more complex systems than quantum microparticles, so the different degree of complexity is an obstacle, to say the least, for accepting the absolute congruence between humans and particles. Quantum physics works on a lower analytical level in comparison to sociology, anthropology, or psychology. Interestingly, humans, as highly complex systems, show some emergent properties, for example, memory and the conscious impression of the continuous persistence of their body and identity across time. When thinking about three different ways of understanding human beings in the quantum perspective, i.e. the realist approach, the functional analogy, and metaphor, one may criticize the realist approach merely because of the fundamental differences in the systems' complexities. Can we compare the behavior of microparticles and humans? Is it possible to use the principles that originated in quantum mechanics in anthropology? Is it possible to use some patterns of the behavior of systems on a lower analytical level for the explanation of the behavior of systems on a higher analytical level? Indeed, these questions are questions challenging the legitimacy of isomorphism across different analytical frames.

General systems theory has used the principle of isomorphism with success for decades. Isomorphism is the equivalence of general form. So there are no one-to-one correspondences. 
The ideas of isomorphism and structural uniformity are accepted by general systems theories and complexity science. However, a key aspect of the transfer of principles between different analytical level is that isomorphic patterns are not absolute one-to-one correspondences, but abstractions. Generalization (or abstraction) is always needed for distinguishing isomorphic patterns. A sufficient level of abstraction is the tool that makes the identification of isomorphic patterns across different levels of analysis possible. There are several steps. The investigator first distinguishes a principle, then abstracts the principle to get a general form, and then uses this generalized principle for the explanation of the behavior of a system on a different level of analysis. This way seems to be also a promising approach for the application of quantum principles in the investigation of human beings. In this perspective, the application of quantum principles on human beings is not realist, but rather a kind of analogy. So, if we use isomorphism, we work with a kind of functional analogy. Of course, many problems still remain and should be examined in future theory developments.

\section{The Problem of the Continuity of Existence}

Quantum mechanics and anthropology differ in the understanding of the permanence of existence. In anthropology, it is assumed that the existence of human beings is relatively permanent during human life. In contrast, quantum theory implies that particles exist only when they are observed. When they are not observed, their existence is described by their wave function, i.e. by the distribution of probabilities.

The Copenhagen interpretation of quantum mechanics suggests that the emergence of an entity is related to the moment of wave function collapse. The wave function of an entity describes the distribution of probabilities with which this entity can be found in a given point of space and time. Wave function collapse is a jump in the state of a particle that occurs in the moment of measurement. In the wave function collapse, one of the possible quantum states is actualized in time and space, and an appearance of existence is available to the senses or apparatus of the human observer. How does a wave function collapse proceed? Before wave function collapses occur, the wave function spreads out over the entire space. Each constituent of matter or energy does not have a clearly defined position in this phase. All things have the properties of a wave in the situation of non-collapsed wave function. At the moment that an observer observes the wave, it peaks and the wave collapses in the sense that it actually has null values in all other places of the space where the given entity was not observed. At the same moment, the observer starts to perceive the observed, e.g. the human body. The chain of wave function collapses ends at the moment when the observer stops their observations and turns their attention to another part of reality. After that, the wave function spreads out over the entire space again.

The concept of wave function collapse can explain the transient existence of microparticles. But can we apply the wave function collapse to the existence human beings? There are several difficulties, because we must consider two different analytical positions. The position of an external observer and a subjective experience of existence by a subject itself. When adopting the position of external observer, I can imagine that a visually impaired person will come to a classroom, and they will not be able to distinguish how many or what people are there. In this case, the quantum logic and the wave function collapse can be applied. From the perspective of an external observer, the existence of people sitting in the classroom is dependent on the act of observation.

However, a different situation occurs when we consider a subjective perspective. We do not know if microparticles have some simple consciousness or simple memory, but human beings have consciousness and memory. These properties enable us to be aware of our identities. We are consciously aware who we are, and we are consciously aware who we are even in those 
momentswhen we are not observed by any external observer. So when considering the position of a conscious human subject, the application of the wave function collapse is problematic.

Wendt's (2015) application of wave function collapse in understanding human beings has been recently criticized by Michel (2018). The problem is how to accept the discontinuity that may be an implication of Wendt's (2015) application of wave function collapse to the interpretation of human beings' existence. If human beings may exist only during the moments of wave function collapse, then the continuity of the existence of human beings is questioned. As a fact, the human subject appears at the moment of wave function collapse as an actualization from the realm of potentialities. The wave function collapse does not suppose a continuity and casual chain of events. But indeed, human brains can maintain a state of quantum coherence and human beings are able to be aware of their identities across longer time periods, at least when they are in normal states of consciousness. Humans have memory, enabling them to remember their identities, as well as previous life events, experiences, etc. This paradox related to the different explanations of the continuity of existence posits several very insistent questions.

\section{CONCLUSION: The Paradox of the Discontinuous Human Experience of Quantum Spacetime}

When considering the human experience of spacetime, we have to accept that the experience of one's identity is not permament all the time. We should realize that the human experience of spacetime continues only during so-called normal states of consciousness. During these states, our identity and location in space and time are relatively stable. But under so-called altered states of consciousness, for example during the flow experience, the situation is very different. During the natural moments of flow, a person experiences being completely absorbed while doing an activity. This absorption is accompanied by the loss of one's sense of space and time. Similar experiences may occur also during other altered states of consciousness, e.g. trance states, experiences during meditation, or during intoxication by psychoactive drugs. There are even instances when a subject can lose its awareness of identity even for longer time periods, e.g. in the case of amnesia caused by brain damage, or amnesia accompanying some mental disorders. All these examples support the assumption that the human experience of identity is discontinuous.

Keeping the discontinuous nature of human experience of spacetime in mind, one may ask how identity is restored after those moments when a different identity was experienced. The reliable answer definitely needs new empirical evidence and research, but at present, at least the principle of a general mechanism for identity restoration can be conceptualized. Interestingly, our memory is able to restore our identity after those moments when our identity is out of awareness. After a period of an altered state of consciousness, the normal state of consciousness is restored, and a subject becomes conscious of who they are again. This process of becoming aware of identity is connected to the restoration of access to a part of memory where the information about one's identity is stored. In a systems perspective, this shows that the presence and maintenance of some kind of relatively stable memory is used for the restoration of a system's identity after periods of the temporary loss of this system's identity.

Furthermore, a subject's memory is also crucial for the development and maintenance of social bonds, and consequently also of social structure. By remembering previous social interactions with other social agents, a subject is able to maintain or terminate its social bonds. A subject's memory is, therefore, an important factor influencing the coherence of social groups, as well as bigger social aggregates. 


\section{REFERENCES}

Allan, B. B. (2018). Social action in quantum social science. Millennium, 47(1), 87-98.

Arfi, B. (2018). Challenges to a quantum-theoretic social theory. Millennium, 47(1), 99-113.

Burgess, J. P. (2018). Science blurring its edges into spirit: The quantum path to Ātma. Millennium, 47(1), 128-141.

Kessler, O. (2018). The mind-body problem and the move from supervenience to quantum mechanics. Millennium, 47(1), 74-86.

Michel, T. (2018). Of particles and humans: The question of 'human being' in Alexander Wendt's Quantum Mind and Social Science. Millennium, 47(1), 114-127.

Russell, H. A. (2013). Quantum Anthropology: Reimaging the human person as body/spirit. Theological Studies, 74, 934-959.

Trnka, R., \& Lorencova, R. (2016). Quantum anthropology: Man, cultures, and groups in a quantum perspective. Prague: Charles University Karolinum Press.

Available at:

https://www.researchgate.net/publication/308792484_Quantum_Anthropology_Man_Cultures _and_Groups_in_a_Quantum_Perspective

Wendt, A. (2015). Quantum mind and social science: Unifying physical and social ontology. Cambridge: Cambridge University Press. 